\title{
Control theory application in power market stability analysis
}

\author{
Youfei Liu, Y.X.Ni, Senior Member, IEEE, and Felix F. Wu, Fellow, IEEE
}

\begin{abstract}
This paper proposes a new framework to model the system dynamics and study the system stability for the quantity bidding competition in power market. From the viewpoint of control theory, the quantity bidding competition is modeled as close-loop dynamic system, where market price is the system feedback signal. Based on the objective of maximizing individual profit in a shot-game, a general dynamic process is given to describe supplier's adjustment behavior, which can be used to model all classical bidding strategy and $\mathrm{CV}$-based bidding strategy. With the application of control theory, sufficient stability conditions and market equilibrium are presented. Computer test results support the analytic conclusion very well.
\end{abstract}

Index Terms: Market dynamics and stability, quantity bidding competition, Power market, One-order inertia system, Negative feedback control system.

\section{INTRODUCTION}

$\mathbf{M}$ ARKET dynamics and stability have long been studied by economists. Generally, an economic system is dynamic if its behavior over time is determined by functional equations in which certain variables interact in an essential way with respect to time horizon. Given the dynamic processes, if they converge to some equilibrium position, then we conclude that it is stable. To model economic dynamics, differential or difference equations are widely adopted, thus the corresponding analysis is based on those differential/difference equations ${ }^{[1][7][8][9]}$.Traditional analysis methods are eigenvalue technique, phase diagrams, Liapunov's direct method, etc. Recently, the application of game theory has drawn much research interest, especially in the competition game modeled by differential equations $^{[10]}$. But till now, there is still no systematic way to study the market stability, and the market equilibrium with multi-players is still difficult to solve with strategic bidding impacts considered .

Work supported by RGC of HK government and China National Key Basic Research.

Youfei Liu is with the Department of Electrical \& Electronic Engineering, Hong Kong University, Hong Kong.(e-mail youfei@eee.hku.hk)

Y.X.Ni is with the Department of Electrical \& Electronic Engincering, Hong Kong University, Hong Kong.(e-mail: yxni@eee.hku.hk)

Felix.F Wu is with the Department of Electrical \& Electronic Engineering, Hong Kong University, Hong Kong.(e-mail: ffwu@eee.hku.hk)
In recent years, competitions have been introduced to power industry in order to improve social welfare and market efficiency. The real power markets are usually oligopolistic and individual suppliers will conduct strategic biddings to seek maximum profit ${ }^{[14]}$. Therefore, it is an important task to study the power market dynamic behavior in time domain and analyze the power market stability and equilibrium.

In the area of analysis of power market dynamics and stability, there has been little prior work. The first work came up in [2], where differential/algebraic equations and eigenvalue techniques are used to analyze the power market stability. It is found that the stability is essentially determined by the differential equation parameters, i.e., the suppliers' cost function parameters and the consumers' utility function parameters. But [2] did not make further study on bidding strategies and their impacts on market dynamics and stability. In [3], the market or bidding process is modeled as a closed-loop dynamic system in which previous and current information are used as a feedback signal. But it is based on a heuristic method, and the given strategy is simple and non-general. In [4], the dynamics and mechanisms of bidding process are investigated. The dynamic model and system objectives are interpreted as a classical control problem. It is found that by introducing multiple bidding rounds, convergence to a Pareto optimum is possible if a PI control policy is introduced. However it considers a very special market situation, thus the corresponding results is not valid in a general case.

In this paper, for a general quantity bidding competition in power market, we propose a new framework to model the market dynamics and analyze the market stability. The basic idea is to model the competition process as a close-loop dynamic system, where the public known information (i.e., the price) is the system feedback signal, and the individual outputs are control variables. Based on the maximizing-profit principle in economics, a general dynamic adjustment rule is defined, which can be used to model various classical bidding strategy (such as perfect competition, Cournot competition, Stackelberg competition, etc) and conjectural variation(CV)based competition. It is shown that different bidding strategies can be modeled as one-order inertia 
control blocks, and the system stability and equilibrium is analyzed using conventional control theory with bidding strategy impacts considered.

This paper is arranged as follows. Section 2 presents a general formulation of quantity bidding competition in power market, and the case of perfect competition bidding strategy is used as an example first. Section 3 studies different kinds of bidding strategies and their impacts to market stability and equilibrium. Numerical tests are given in the section 4 with conclusions drawn in section 5 .

\section{POWERR MARKET DYNAMIC MODEL FORMULATION}

In order to make power market stability study, a general purpose power market dynamical model is formulated in this section, which is to be extended in section 3 for considering various bidding strategy impacts to power market stability and equilibrium. Basic assumptions made in this paper are as follows: there are $n$ suppliers (generators) in power market with quadratic production cost function competing to each other in spot market to maximize their profit while serving the demand; the demand curve is linear and steady with respect to time (i.e., we only study the same hour of any day successively); electricity can not be stored, thus the power supply and demand are always balanced; and individual production cost functions are private and unknown to others; while the market clearing price is publicly known; generator $i(i=1, \cdots n)$ needs to decide $q_{i}$ with respect to available market price $p$ with certain time delay (i.e., the repeated biddings are considered as continuous events); generator capacity limits and network effects are neglected at this stage.

Suppose the cost function of supplier (or generator) $i$ is quadratic and the studied time period is one-hour, i.e:

$$
C_{i}\left(q_{i}\right)=a_{i}+b_{i} q_{i}+\frac{1}{2} c_{i} q_{i}^{2}, \quad i=1, \cdots, n
$$

where $q_{i}$ : power output of supplier $i ; a_{i}, b_{i}, c_{i}$ : parameters of supplier $i$ 's cost function. The corresponding marginal cost function will be:

$$
C_{i}^{\prime}\left(q_{i}\right)=b_{i}+c_{i} q_{i}, \quad i=1, \cdots, n
$$

Assume that the electricity demand is linear and the corresponding inverse demand curve takes the form:

$$
p=F(D)=e-f D=e-f \sum_{i=1}^{n} q_{i}
$$

where $D$ : total demand; $e, f:$ coefficients can be derived from historical records and publicly known, and $e>0, f>0$; total supply $Q=\sum_{i=1}^{n} q_{i}=D$. Thus for a specific total supply $Q$, the market clearing price $p$ is determined by equation (3).
Supplier $i$ 's profit function is defined by:

$$
\pi_{i}=p q_{i}-C_{i}\left(q_{i}\right)
$$

In the market, each supplier will make its optimal decision on $q_{i}$ to maximize its profit based on market information. We assume supplier's dynamic response can be described by ${ }^{[11]}$ :

$$
\frac{d q_{i}}{d t}=\lambda_{i} \frac{\partial \pi_{i}}{\partial q_{i}}, \lambda_{i}>0
$$

where $\lambda_{i}$ is a factor representing the speed of adjustment or the preference of adjustment intensity. Equation (5) indicates that suppliers will adjust their outputs in the direction of increasing profit.

If a power market is perfectly competitive, price $p$ in (4) is independent to individual $q_{i}$, the stability of perfect competition market is easy to analyze. However the real power market is usually oligopolistic and price $p$ is dependent to $q_{i}(i=1, \cdots, n)$. As we all know in power markets that $q_{i}$ is private information, and individual suppliers will conduct strategic biddings in real oligopolistic power markets. As a result, market stability is of great concern and its analysis is nontrivial. In this paper, market dynamic model under perfect competition will be derived first. It will then be modified to meet different bidding strategies so as to study their impacts on market dynamics.

In perfect competition, no one can influence the market price, i.e., $\partial p / \partial q_{i}=0$. Based on equations (1) and (4), we know:

$$
\frac{\partial \pi_{i}}{\partial q_{i}}=p-\left(b_{i}+c_{i} q_{i}\right)=\left(p-b_{i}\right)-c_{i} q_{i}
$$

And the first order condition for maximizing the profit is given by:

$$
\frac{\partial \pi_{i}}{\partial q_{i}}=0
$$

Defining an apparent 'price' (a coordinate shift to simplify the notation):

$$
p_{i}=p-b_{i}
$$

Substituting (6a) into (5), supplier $i$ 's dynamic adjustment rule is:

$$
\frac{d q_{i}}{d t}=\lambda_{i}\left[p_{i}-c_{i} q_{i}\right] \lambda_{i}>0, i=1, \cdots, n
$$

By Laplace transformation, the corresponding transfer function of (8) takes the form:

$$
G_{i}(S)=\frac{q_{i}(S)}{p_{i}(S)}=\frac{\frac{1}{c_{i}}}{1+\frac{1}{\lambda_{i} c_{i}} S}=\frac{K_{i}}{1+T_{i} S}, i=1, \cdots, n
$$

Equations (8) and (9) show that the supplier strategy in a perfect competition spot market corresponds to a first-order inertia control system with gain and time constant taken as: 


$$
\left\{\begin{array}{l}
K_{i}=\frac{1}{c_{i}} \\
T_{i}=\frac{1}{\lambda_{i} c_{i}}
\end{array}\right.
$$

It is reasonable that larger $\lambda_{i}$ corresponds to smaller time constant, i.e., faster adjustment; and that larger $c_{i}$, which means faster cost raising along with output increasing, will lead to smaller $K_{i}$ and $T_{i}$. The system block diagram for perfect competition is shown in Fig. 1.

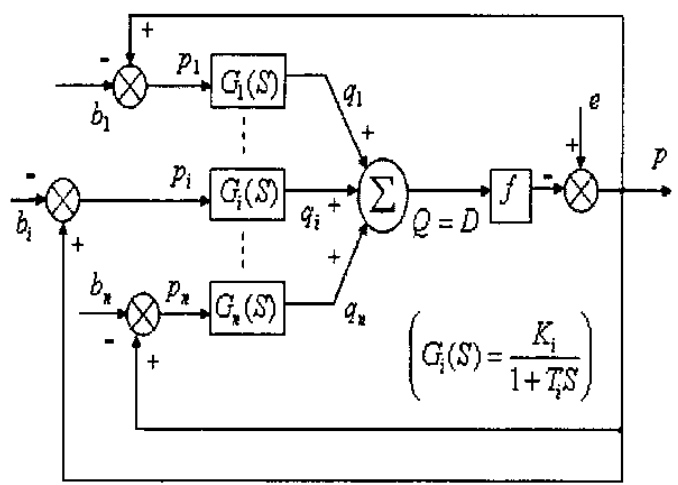

Fig. 1. System diagram for dynamic adjustment process

From control theory, the system will be stable if all the poles of the system close-loop transfer function have negative real parts, which is equivalent to that all the system eigenvalues have negative real parts using state-space equation notation. In order to simplify global system (See Fig. 1) stability, we introduce the following proposition.

Proposition: For a system with first-order open-loop transfer functions shown in Fig. 1, if all open-loop transfer functions are stable, then the close-loop system is stable. (See Appendix A for proof.)

From equation (9), the open-loop system poles (See Fig. 1) are given by:

$$
1+S_{i} T_{i}=0 \text { or } \mathrm{S}_{i}=-\lambda_{i} c_{i}, \quad i=1, \cdots, n(11)
$$

Hence, the perfect competition market stability condition is simply $\left(\because \lambda_{i}>0\right)$ :

$$
S_{i}<0 \text { or } c_{i}>0, i=1, \cdots, n
$$

Assuming that $c_{i}>0(i=1, \cdots, n)$ and according to (3) and (6), the market clearing price at individual optimum decisions or system equilibrium $\left(\because \frac{d q_{i}}{d t}=\lambda_{i} \frac{\partial \pi_{i}}{\partial q_{i}}=0, \quad i=1, \cdots, n\right)$ will be:

$$
p^{*}=e-f \sum_{i=1}^{n} q_{i}^{*}=e-f \sum_{i=1}^{n} \frac{p^{*}-b_{i}}{c_{i}}
$$

$p^{*}$ can be solved from (13) and the system stable equilibrium is:

$$
p^{*}=\frac{e+f \sum_{i=1}^{n} \frac{b_{i}}{c_{i}}}{1+\sum_{i=1}^{n} \frac{f}{c_{i}}}, q_{i}^{*}=\frac{p^{*}-b_{i}}{c_{i}}(i=1, \cdots, n)
$$

Fig. 1 and (14) show that although supplier $i \quad(i=1, \cdots n)$ does not know his rival's $\left(b_{j}, c_{j}\right)(j \neq i)$, so far as he behaves according to (5) in a perfect competition market, the market will be stable and converge to $\left(p^{*}, q^{*}\right)$, where $q^{*}=\left(q_{1}^{*}, \cdots, q_{n}^{*}\right)^{T}$.

It should be pointed out that Fig. 1 is a general purpose system diagram for power markets. Actually different kinds of transfer function $G_{i}($.$) can be used$ to simulate various bidding strategies in oligopolistic power markets and their impacts on power market stability can be studied in detail. This is done in the next section.

\section{POWER MARKET STABILITY ANALYSIS WITH DIFFERENT BIDDING STRATEGIES}

\section{A. Cournot competition case}

The real power markets are usually oligopolistic rather than perfect competition, where a few suppliers might have noticeable market shares. Strategic biddings are utilized to maximize individual profits. Among various bidding strategies, Cournot competition model is widely used. Under Cournot competition, each supplier makes his decision assuming others have certain outputs, i.e., $\partial q_{j} / \partial q_{i}=0(j \neq i)$. Therefore, $\partial \pi_{i} / \partial q_{i}$ is $(\because$

$$
\begin{aligned}
\partial p / \partial q_{i} & \left.=-f \text { and } p=e-f \sum_{i=1}^{n} q_{i}\right): \\
& \frac{\partial \pi_{i}}{\partial q_{i}}=\frac{\partial}{\partial q_{i}}\left[p q_{i}-\left(a_{i}+b_{i} q_{i}+\frac{1}{2} c_{i} q_{i}^{2}\right)\right] \\
& =\left(p-b_{i}\right)-\left(f+c_{i}\right) q_{i}, \quad i=1, \cdots, n
\end{aligned}
$$

and from (5) the dynamic adjustment rule will be $\left(p_{i}=p-b_{i}\right)$ :

$$
\frac{d q_{i}}{d t}=\lambda_{i}\left[p_{i}-\left(f+c_{i}\right) q_{i}\right] \quad \lambda_{i}>0, \quad i=1, \cdots, n
$$

Based on (16), we can obtain the following individual transfer function for Cournot competition as:

$$
\begin{aligned}
& G_{i}(S)=\frac{q_{i}(S)}{p_{i}(S)}=\frac{\lambda_{i}}{S+\lambda_{i}\left(f+c_{i}\right)} \\
& =\frac{\frac{1}{\left(f+c_{i}\right)}}{1+\frac{1}{\lambda_{i}\left(f+c_{i}\right)} S}=\frac{K_{i}^{c}}{1+T_{i}^{c} S}, \quad i=1, \cdots, n
\end{aligned}
$$

As compared with perfect competition, the only difference is $c_{i}$ in (9) is replaced by $\left(f+c_{i}\right)$ in (17) 
to include $q_{i}$ 's impact on price $p$, and hence on the profit $\pi_{i}$. The system diagram is the same as Fig. 1 .

Based on the proposition in the paper, the sufficient condition for power market stability of Cournot competition will be simply $\left(\because \lambda_{i}>0\right)$ :

$$
f+c_{i}>0, \quad i=1, \cdots, n
$$

Usually $f>0,(18)$ is surely satisfied if $c_{i}$ is positive.

Similarly the stable equilibrium of the system can be solved for as:

$$
p^{*}=\frac{e+f \sum_{i=1}^{n} \frac{b_{i}}{c_{i}+f}}{1+\sum_{i=1}^{n} \frac{f}{c_{i}+f}}, q_{i}^{*}=\frac{p^{*}-b_{i}}{c_{i}+f}(i=1, \cdots, n)
$$

\section{B. Stackelberg competition case}

In Stackelberg competition, there are two different types of suppliers, i.e. leaders and followers. For convenience, let supplier 1 be the leader and the others the followers. For followers, it can be proven ${ }^{[12]}$ that their behaviors are similar to firms in Cournot competition and taking $\partial q_{j} / \partial q_{i}=0$ ( $i \in$ followers $)$. Thus, the following sufficient conditions for system stability should be satisfied:

$$
f+c_{i}>0, \quad i=2, \cdots, n
$$

For the leader, he knows that the followers will react to his strategy similar to Cournot competition, therefore $\partial \pi_{1} / \partial q_{1}$ can be derived as

$$
\begin{aligned}
& \left(p=e-f \sum_{j=1}^{n} q_{j}\right): \\
& \quad \frac{\partial \pi_{1}}{\partial q_{1}}=\left(p-b_{1}\right)+\left(\frac{\partial p}{\partial q_{1}}-c_{1}\right) q_{1}
\end{aligned}
$$

where $\frac{\partial p}{\partial q_{1}}=-f-f \sum_{i=2}^{n} \frac{\partial q_{i}}{\partial q_{1}}$ and

$$
\sum_{i=2}^{n} \frac{\partial q_{i}}{\partial q_{1}}=\frac{\sum_{i=2}^{n} \frac{f}{f+c_{i}}}{1+\sum_{i=2}^{n} \frac{f}{f+c_{i}}}(\text { See Table } 2 \text { in [6]). }
$$

Substituting (21) into (5), dynamic adjustment rule for supplier 1 as a leader in Stackelberg competition is given by $\left(p_{1}=p-b_{1}\right)$ :

$$
\frac{d q_{1}}{d t}=\lambda_{1}\left[p_{1}-\left(f+f \frac{\sum_{i=2}^{n} \frac{f}{f+c_{i}}}{1+\sum_{i=2}^{n} \frac{f}{f+c_{i}}}+c_{1}\right) q_{1}\right], \lambda_{1}>0
$$

Based on the proposition in the paper, the corresponding sufficient condition for market stability of the leader can be derived similar to (16) and (17) of Cournot competition as $\left(\because \lambda_{1}>0\right)$ :

$$
f+f \frac{\sum_{i=2}^{n} \frac{f}{f+c_{i}}}{1+\sum_{i=2}^{n} \frac{f}{f+c_{i}}}+c_{1}>0
$$

In terms of (20) and (23), the sufficient conditions for integrated power market stability are:

$$
\left\{\begin{array}{c}
f+f \frac{\sum_{i=2}^{n} \frac{f}{f+c_{i}}}{1+\sum_{i=2}^{n} \frac{f}{f+c_{i}}}+c_{1}>0 \\
f+c_{i}>0, i=2, \cdots, n
\end{array}\right.
$$

Usually $f>0$ and $c_{i}>0(i=1, \cdots, n)$, hence (24) can be satisfied and the market is stable. The stable equilibrium can be derived without difficulty:

$$
\left\{\begin{array}{c}
p^{*}=\frac{e+f \sum_{i=2}^{n} \frac{b_{i}}{c_{i}+f}+f b_{1} \mu}{1+\sum_{i=2}^{n} \frac{f}{c_{i}+f}+f \mu} \\
q_{i}^{*}=\frac{p^{*}-b_{i}}{c_{i}+f}(i=2, \cdots, n), q_{1}^{*}=\left(p^{*}-b_{1}\right) \mu
\end{array}\right.
$$

where $\mu=\left(c_{1}+f+f \frac{\sum_{i=2}^{n} \frac{f}{f+c_{i}}}{1+\sum_{i=2}^{n} \frac{f}{f+c_{i}}}\right)^{-1}$.

C. Bidding strategy based on conjectural variation concept

\section{Constant CV Case}

Another kind of popular bidding strategy is based on conjectural variation ${ }^{[5][6]}$. The conjectural variation is defined as one's belief or expectation on its rival's reaction to its output changes. For supplier $i$, his conjecture on supplier $j$ 's variation as a react to its output change is:

$$
C V_{i j}=\frac{\partial q_{j}}{\partial q_{i}}
$$

A useful concept is the integrated response of supplier $i$ 's rivals:

$$
C V_{-i}=\sum_{j=1, j \neq i}^{n} C V_{i j}=\sum_{j=1, j \neq i}^{n} \frac{\partial q_{j}}{\partial q_{i}}
$$

Assuming $C V_{-i}$ can be observed in terms of power market historical records and $C V_{-i}$ is constant, 


$$
\begin{gathered}
\partial \pi_{i} / \partial q_{i} \text { of supplier } i \text { will be }\left(p=e-f \sum_{i=1}^{n} q_{i}\right) \\
\frac{\partial \pi_{i}}{\partial q_{i}}=p-f\left(1+\sum_{j=1, j \neq i}^{n} \frac{\partial q_{j}}{\partial q_{i}}\right) q_{i}-\left(b_{i}+c_{i} q_{i}\right) \\
=\left(p-b_{i}\right)-\left(f+f C V_{-i}+c_{i}\right) q_{i}, \quad i=1, \cdots, n
\end{gathered}
$$

The corresponding dynamic adjustment rule is:

$\frac{d q_{i}}{d t}=\lambda_{i}\left[p_{i}-\left(f+f C V_{-i}+c_{i}\right) q_{i}\right] \quad \lambda_{i}>0, \quad i=1, \cdots, n$

The individual transfer functions in Fig. 1 can be derived based on (29) as:

$$
\begin{aligned}
& G_{i}(S)=\frac{q_{i}(S)}{p_{i}(S)}=\frac{\frac{1}{f+f C V_{-i}+c_{i}}}{1+\frac{1}{\lambda_{i}\left(f+f C V_{-i}+c_{i}\right)} S} \\
& =\frac{K_{i}^{c v} .}{1+T_{i}^{c v} S}, \quad i=1, \cdots, n
\end{aligned}
$$

and the sufficient stability condition for the power market is:

$$
f+f C V_{-i}+c_{i}>0, \quad i=1, \cdots, n
$$

The system equilibrium is given by:

$$
\left\{\begin{array}{c}
p^{*}=\frac{e+f \sum_{i=1}^{n} \frac{b_{i}}{f+f C V_{-i}+c_{i}}}{1+\sum_{i=1}^{n} \frac{f}{f+f C V_{-i}+c_{i}}} \\
q_{i}^{*}=\frac{p^{*}-b_{i}}{f+f C V_{-i}+c_{i}},(i=1, \cdots, n)
\end{array}\right.
$$

It can be proven ${ }^{[6][12]}$ that classical game theoretical bidding strategies are corresponding to specific $C V_{-i}$ values. For example, $C V_{-i}=-1$ corresponds to perfect competition strategy; and $C V_{-i}=0$ corresponds to Cournot competition strategy, and $C V_{-i}=c_{i} \sum_{j \neq i} \frac{1}{c_{j}}$ corresponds to collusion $^{[13]}$. For a rational supplier, his bidding strategy should be in-between perfect competition and collusion. Therefore we have $-1 \leq C V_{-i} \leq c_{i} \sum_{j \neq i} \frac{1}{c_{j}}$ and the sufficient stability condition (31) can be $c_{i}>0(i=1, \cdots, n)$ since $f+f C V_{-i} \geq 0$.

\section{Dynamic CV Case}

When $C V_{-i}$ evolves with respect to time, i.e., $C V_{-i}$ is dynamic, a key issue for a supplier is how to make adaptive estimation of $\mathrm{CV}_{-i}$ using available market data. Define dynamic conjecture variation as follows:

$$
C V_{-i}=\sum_{j \neq i} \frac{d q_{j}}{d q_{i}}=\frac{\sum_{j \neq i} d q_{j}}{d q_{i}}=\frac{d q_{-i}}{d q_{i}}
$$

where ' $d q_{-i}$ ' is a rivals' integrated differential with respect to the differential of ' $d q_{i}$ '. From equation (3), we can obtain:

$$
d p_{i}=d p=-f d Q=-f \sum_{i=1}^{n} d q_{i}=-f d q_{i}-f d q_{-i}
$$

or

$$
d q_{-i}=-\frac{d p_{i}}{f}-d q_{i}
$$

Then

$$
C V_{-i}=\frac{d q_{-i}}{d q_{i}}=-\frac{1}{f} \frac{\dot{p}_{i}}{\dot{q}_{i}}-1=\psi_{i}\left(\dot{q}_{i}, \dot{p}_{i}\right)
$$

It is easy to prove that under the new $\mathrm{CV}$ definition (33), and for the extreme case of perfect competition, the $C V_{-i}$ is still equal to $(-1)$ ( $\because \frac{d p_{i}}{d q_{i}}=0$ for perfect competition). It is clear that a rational supplier will take dynamic $C V_{-i} \geq-1$.

The system diagram for adaptive $\mathrm{CV}$-based competitive power market is shown in Fig. 2 with $G_{i}(S)$ and $\psi_{i}\left(\dot{q}_{i}, \dot{p}_{i}\right)$ defined in (30) and (36) respectively.

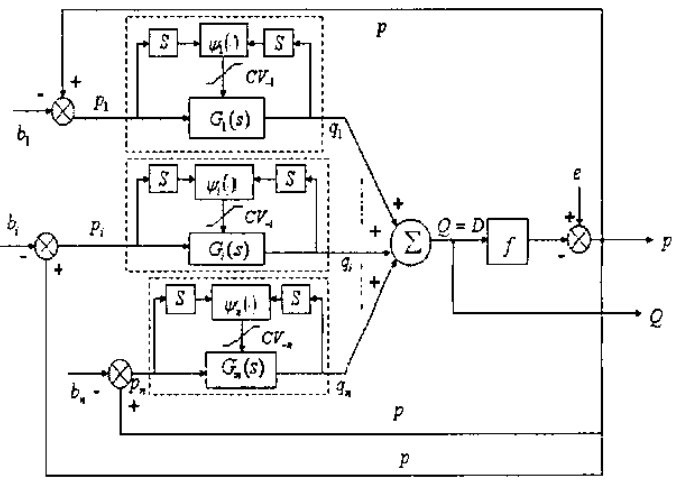

Fig. 2. System diagram with adaptive conjecture competition

In adaptive $\mathrm{CV}$-based competition, although the transfer function parameters $\left(K_{i}, T_{i}\right)$ are functions of dynamic $C V_{-i}$ and time-varying, for a specific instantaneous $C V_{-i}(\geq-1)$, each supplier's control system is still a first-order system. As long as the open-loop system poles are negative all the time, the system will keep stable. Therefore the sufficient condition for system stability is still $\left(\because C V_{-i} \geq-1\right)$ :

$$
f+f C V_{-i}+c_{i}>c_{i}>0, \quad i=1, \cdots, n
$$

It is easy to know that when $C V_{-i}$ moves off from negative unity towards positive, the system will become more and more stable, which means that Cournot competition (where $C V_{-i}=0$ ) is 
more stable than perfect competition (where $\left.C V_{-i}=-1\right)$ in the sense of short time period of price transients under same disturbances.

\section{NUMERICAL RESULTS}

The IEEE 6-generator 30-bus system is used to demonstrate the proposed method. The supplier parameters (including the cost function coefficients and dynamic adjustment parameters) are listed in Table 1.

TABLE I

Cost function coefficients and dynamic adjustment parameters

\begin{tabular}{|c|c|c|c|c|}
\hline Supplicr & $\lambda_{i}$ & $a_{i}$ & $b_{i}$ & $c_{i}$ \\
\hline 1 & 3 & 0 & 2 & 0.02 \\
\hline 2 & 4 & 0 & 1.75 & 0.0175 \\
\hline 3 & 2.5 & 0 & 3 & 0.025 \\
\hline 4 & 3 & 0 & 3 & 0.025 \\
\hline 5 & 2 & 0 & 1 & 0.0625 \\
\hline 6 & 4 & 0 & 3.25 & 0.00834 \\
\hline
\end{tabular}

Assume that the inverse demand function is known as:

$$
p=50-0.02 D=50-0.02 \sum_{i=1}^{6} q_{i}
$$

\section{A.Test case $1:$ perfect competition}

Table 2 illustrates the system equilibrium and eigenvalues for perfect competition. The system eigenvalues are calculated through state space equation (See appendix ). As all the eigenvalues are negative, the system is stable, which can also be verified by the stability condition (13). The corresponding system equilibrium is solved from (14).

TABLE II

Equilibrium and dynamic characteristics under perfect competition $\left(p^{*}=8.9685 \$ / \mathrm{MWh}\right)$.

\begin{tabular}{|c|c|c|c|}
\hline Supplicr & $q_{i}^{*}(\mathrm{MW})$ & $\pi_{i}^{*}(\$ / \mathrm{h})$ & $\begin{array}{l}\text { Eigen - } \\
\text { values }\end{array}$ \\
\hline $\bar{l}$ & 348.43 & 1214 & \multirow{7}{*}{$\begin{array}{l}-0.4380 \\
-0.1171 \\
-0.0405 \\
-0.0612 \\
-0.0659 \\
-0.0732\end{array}$} \\
\hline 2 & 412.49 & 1488.8 & \\
\hline 3 & 238.74 & 712.5 & \\
\hline 4 & 238.74 & 712.5 & \\
\hline 5 & 127.49 & 508 & \\
\hline 6 & 685.67 & 1960.5 & \\
\hline$\sum$ & 2051.6 & 6596.3 & \\
\hline
\end{tabular}

Given any initial condition, we can simulate the system time response. Fig. 3 shows the time response with the initial condition $q_{i}(0)=0$ $(i=1, \cdots, n)$, where time unit is fictitious and used only for observations of $q_{i}$ 's evolution.

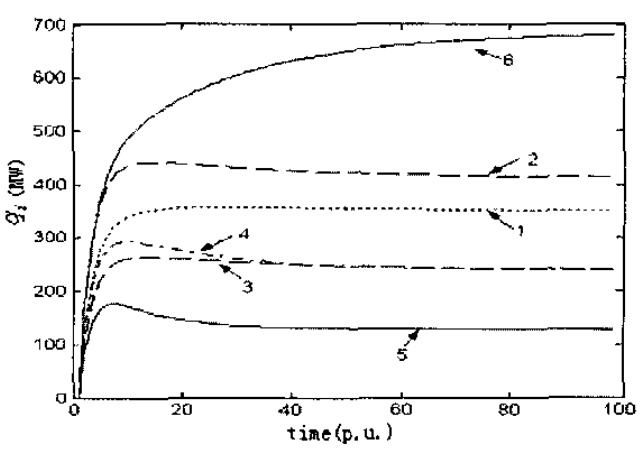

Fig. 3. Time response of suppliers' outputs under perfect compctition

It should be pointed out that the dynamic adjustment parameter $\lambda_{i}$ will not influence the system stability, but it will affect the time response, as shown in Fig. 3, where suppliers 3 and 4 have the same cost function coefficients with different adjustment parameters $\left(\lambda_{3}=2.5, \lambda_{4}=3\right)$, and supplier 4 has a faster initial rising in output than supplier 3.

\section{B. Test case 2: Cournot competition}

Under Cournot competition, all suppliers' $C V_{-i}$ are held as constant 0 . Table 3 shows the equilibrium and eigenvalues for Cournot competition. As all the eigenvalues are negative, the system is stable. Comparing the results with Table 2, we know that the system eigenvalues under Cournot competition are more negative, which means the market is more stable.

TABLE III

Equilibrium and dynamic characteristics under Coumot competition $\left(p^{*}=14.76 \$ / \mathrm{MWh}\right)$.

\begin{tabular}{|c|c|c|c|}
\hline Supplier & $\begin{array}{c}q_{i}^{*} \\
(\mathrm{MW})\end{array}$ & $\begin{array}{c}\pi_{i}^{*} \\
(\$ / \mathrm{h})\end{array}$ & $\begin{array}{l}\text { Eigen } \\
\text {-values }\end{array}$ \\
\hline$I$ & 319.06 & 3054 & \multirow{7}{*}{$\begin{array}{c}-0.5023 \\
-0.1617 \\
-0.1431 \\
-0.1286 \\
-0.1174 \\
-0.1128\end{array}$} \\
\hline 2 & 346.99 & 3461.7 & \\
\hline 3 & 261.38 & 2220.5 & \\
\hline 4 & 261.38 & 2220.5 & \\
\hline 5 & 166.81 & 1426.2 & \\
\hline 6 & 406.22 & 3988.5 & \\
\hline$\sum$ & 1761.8 & 16371 & \\
\hline
\end{tabular}

Fig. 4 shows the time evolution of suppliers' outputs. Comparing to test case 1 of perfect competition, Cournot competition will lead to lower total output and a higher market clearing price, and has a relatively faster response towards equilibrium. Moreover we can see that the individual profits are much higher than in test case 1. 


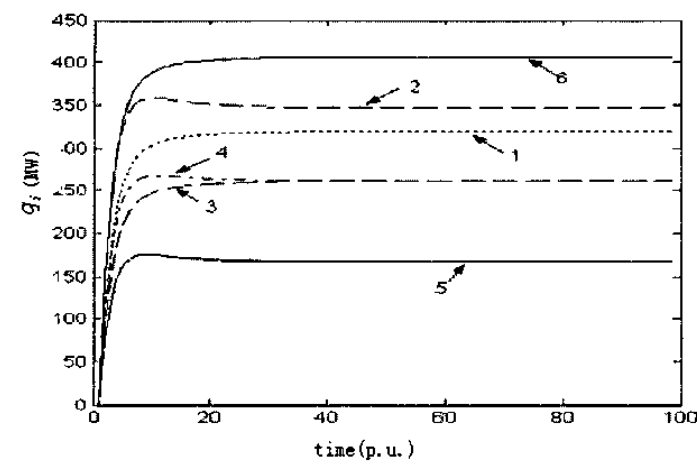

Fig. 4. Time response of suppliers' outputs under Coumot compctition

In the same way, we have simulated more cases such as Stackelberg competition, constant and adaptive CV-based competition, etc. The test results match the analytic conclusions very well. The results will not be presented here.

\section{CONLUSION}

From the viewpoint of control theory, this paper proposes a general framework for modeling market dynamics and analyzing market stability for the quantity bidding competition in power markets. The basic idea is to model the quantity bidding process as a close-loop dynamic system, where market price is the system feedback signal.Based on the maximizing profit principle in economics, this paper suggests a general output adjustment rule, which is suitable for modeling various classical competition strategies and $\mathrm{CV}$-based bidding strategies. Propositions are proved for fast market stability judgment. The sufficient condition and market equilibrium under various bidding strategies are presented in this paper. Numerical test results support the analytic conclusions very well.

\section{APPENDIX}

Proposition 1: For a linear system $A \dot{X}=B X$, if $A$ is a positive definite matrix and $B^{T}=B$, then there exists an invertible matrix $Q$ such that $Q^{T} A Q=I$ and $Q^{T} B Q$ is a diagonal matrix, and the condition for the system to be stable is that matrix $B$ is negative definite.

Proof: Since A is positive definite matrix, then there exists an invertible matrix $Q_{1}$ such that $Q_{1}^{T} A Q_{1}=I$. Consider the symmetry matrix $Q_{1}^{T} B Q_{1}$, we have an orthogonal matrix $U$ such that $U^{T} Q_{1}^{T} B Q_{1} U$ is a diagonal matrix ${ }^{[13]}$. Let $Q=Q_{1} U$, then $Q$ is the required matrix such that $Q^{T} A Q=I$ and $Q^{T} B Q$. Through a linear transformation $X=Q Y$, it yields:

$$
Q^{T} A Q \dot{Y}=Q^{T} B Q Y
$$

Hence:

$$
\dot{Y}=Q^{T} B Q Y
$$

From linear system theory, we know that the linear transformation will not change the property of system stability. Therefore, if system $\dot{Y}=Q^{T} B Q Y$ is stable, the system $A \dot{X}=B X$ is stable too. For system $\dot{Y}=Q^{T} B Q Y$ to be stable, it requires that the symmetrical matrix $Q^{T} B Q$ is negative definite. For matrix $Q^{T} B Q$ to be negative definite, given any vector $U$, it must satisfy the following condition:

$$
U^{T} Q^{T} B Q U<0
$$

Denote a new vector $V=Q U$, we can have:

$$
V^{T} B V<0
$$

That is to say, matrix $B$ should be negative definite.

Q.E.D

Proposition 2: For a matrix taking the form

$$
B=-\left[\begin{array}{llll}
c_{1} & & & \\
& c_{2} & & \\
& & \ddots & \\
& & & c_{n}
\end{array}\right]-f\left[\begin{array}{cccc}
1 & 1 & \cdots & 1 \\
1 & 1 & \cdots & 1 \\
\vdots & \vdots & & \vdots \\
1 & 1 & \cdots & 1
\end{array}\right]
$$

If the following condition is satisfied:

$$
\left\{\begin{array}{l}
f>0 \\
c_{i}>0, i=1, \cdots, n
\end{array}\right.
$$

then the matrix $B$ is negative definite.

Proof: For any nonzero vector $x=\left(x_{1}, x_{2}, \cdots, x_{n}\right)^{\prime}$, we have:

$x^{T} B x=-\sum_{i=1}^{n} c_{i} x_{i}^{2}-f\left(x_{1}+x_{2}+\cdots+x_{n}\right)^{2}<-\sum_{i=1}^{n} c_{i} x_{i}^{2}$

If $c_{i}>0, i=1, \cdots, n$, then it is clear to know that $x^{T} B x<0$.

Q.E.D

Proposition 3: For a system with first-order openloop transfer functions shown in Fig. 1, if all openloop transfer functions are stable, then the closeloop system is stable.

Proof: The system shown by Fig. 1 is a coupled negative feedback control system, in order to study the system stability we model the system with state space equation. Without loss of generality, consider the perfect competition case, thus we have:

$$
\left.\left.\left[\begin{array}{cc}
\left(\lambda_{1}\right)^{-1} & \\
\left(\lambda_{2}\right)^{-1} \\
\ddots \\
\\
\\
\left(\lambda_{n}\right)^{-1}
\end{array}\right] \begin{array}{l}
\dot{q}_{1} \\
\dot{q}_{2} \\
\vdots \\
\dot{q}_{n}
\end{array}\right]=-\left[\begin{array}{lll}
c_{1} & & \\
& c_{2} & \\
& \ddots & \\
& & c_{n}
\end{array}\right] \begin{array}{l}
q_{1} \\
q_{2} \\
q_{n}
\end{array}\right]+\left[\begin{array}{l}
p_{1} \\
p_{2} \\
\vdots \\
p_{n}
\end{array}\right]
$$




$$
\begin{aligned}
& =-\left[\begin{array}{cccc}
\left(f+c_{1}\right) & f & \cdots & f \\
f & \left(f+c_{2}\right) & \cdots f \\
\vdots & \ddots & \vdots \\
f & f & \cdots & f \\
& \left(f+c_{n}\right)
\end{array}\right]\left[\begin{array}{l}
q_{1} \\
q_{2} \\
\vdots \\
q_{n}
\end{array}\right] \\
& +\left[\begin{array}{l}
e-b_{1} \\
e-b_{2} \\
\vdots \\
e-b_{n}
\end{array}\right]=A_{n \times n}\left[\begin{array}{l}
q_{1} \\
q_{2} \\
\vdots \\
q_{n}
\end{array}\right]+\left[\begin{array}{l}
e-b_{1} \\
e-b_{2} \\
\vdots \\
e-b_{n}
\end{array}\right]
\end{aligned}
$$

Rewrite the matrix $A_{n \times n}$ as:

$$
A_{n \times n}=-\left[\begin{array}{cccc}
c_{1} & & & \\
& c_{2} & \\
& & \ddots & \\
& & & c_{n}
\end{array}\right]-f\left[\begin{array}{cccc}
1 & 1 & \cdots & 1 \\
1 & 1 & \cdots & 1 \\
\vdots & \vdots & & \vdots \\
1 & 1 & \cdots & 1
\end{array}\right]
$$

Then, from proposition 1 , the stability of above system requires that matrix $A_{n \times n}$ is negative definite. As $f \geq 0$, from proposition 2 , the sufficient condition for the system given by (A-7) to be stable is:

$$
c_{i}>0
$$

which means that if all the poles of open-loop transfer function are negative, or all the open-loop transfer functions are stable, the close-loop system is stable.

\section{REFERENCE}

Periodicals:

[1] Kenneth J. Arrow, H. D. Block, Leonid Hurwicz, "On the Stability of the Competitive Equilibrium, II", Econometrica, Vol. 27, No. 1. (Jan., 1959), pp. 82-109.

[2] Alvarado.F, "The stability of power system markets", IEEE Transactions on Power Systems, Vol.14,No.2, pp.505 -511, May 1999.

[3] Visudhiphan.P.,Ilic.M.D., "Dynamic games-based modeling of electricity markets", Powcr Engineering Society 1999 Winter Meeting, Vol.1, pp.274 -281, 31 Jan-4 Feb 1999.

[4] Syothert.A,MacLeod.I, "Competitive bidding as a control problem", IEEE Transactions on Power Systems, Vol.15, No.1, pp:88-94 Fcb. 2000.

[5] Morton I. Kamien, Nancy L. Schwartz, "Conjectural variations", The Canadian Journal of Economics, Vol. 16, No. 2. (May, 1983), pp. 191-211.

[6] Y.Q.Song,Y.X.Ni, F.S.Wen, et al, "Conjectural variation based bidding strategy in spot markets: fundamentals and comparison with classical game theoretical bidding strategies", Electric Power systems Research, Volume 67, Issue 1, October 2003, Pages 45-51.

Books:

[7] Samuclson P.A., Foundations of Economic analysis, Harvard University Press, 1947

[8] Giancarlo Gandolfo, Economic dynamics, $3^{\text {rd }}$ edition, Springer, 1996.

[9] Brian Beavis, Ian. M. Dobbs, Optimization and stability theory for economic analysis, Cambridge university press, 1990.

[10] Tamer Basar, Geert Jan Olsder, Dynamic Noncooperative Game Theory, $2^{\text {rd }}$ edition, SIAM, 1995.

[11] Hal R.Varian, Microeconomic Analysis, $3^{\text {rd }}$ edition, W.W.Norton\& Company, 1992.

[12] J.Church, R.Ware, Industrial Organization: A strategic Approach, Irwin McGraw-Hill, Boston, 2000.
[13] Lee W.Johnson, R.Dean Riess, Jimmy T. Amold, Introduction to linear algebra, Prentice-Hall,2000.

Papers from Conference Proceedings (Published):

[14] A.K.David, F.S.Wen, "Strategic bidding in competitive electricity markets: A literature survey", Proceedings of IEEE PES 2000 Summer Power Meeting, Scattle, USA, 2000, pp.2168-2173.

\section{BIOGRAPHIES}

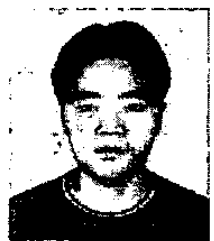

Youfei Liu was born in JiangXi, P.R.C, on Jan 20,1979 . He obtained his master degree from University of Science \&Technology of China in 2002, and now is a PHD candidate of Hong Kong university. His current research interest is the analysis of dynamics and stability of power market 